\title{
Correction to: Seismicity in the block mountains between Halle and Leipzig, Central Germany: centroid moment tensors, ground motion simulation, and felt intensities of two $M \approx 3$ earthquakes in 2015 and 2017
}

\author{
Torsten Dahm • Sebastian Heimann • Sigward Funke • Siegfried Wendt • \\ Ivo Rappsilber · Dino Bindi · Thomas Plenefisch • Fabrice Cotton
}

Published online: 10 August 2018

(C) Springer Nature B.V. 2018

Correction to: J Seismol (2018) 22:985-1003

https://doi.org/10.1007/s10950-018-9746-9

The original version of this article unfortunately contains a mistake. Table 3 in the Appendix A is the preliminary version and is not correctly given. The corrected version of Table 3 is given below.

The online version of the original article can be found at https://doi.org/10.1007/s10950-018-9773-6.

T. Dahm $(\varangle) \cdot$ F. Cotton $\cdot$ S. Heimann · D. Bindi GFZ German Research Centre for Geosciences, Helmholtzstraße 7, 14467 Potsdam, Germany

e-mail: torsten.dahm@gfz-potsdam.de

T. Dahm · F. Cotton

Institute of Earth and Environmental Science,

University of Potsdam, Potsdam, Germany

S. Funke $\cdot$ S. Wendt

Institute for Geophysics and Geology, Leipzig University,

Leipzig, Germany

I. Rappsilber

Landesamt für Geologie und Bergwesen Sachsen-Anhalt,

Halle, Germany

T. Plenefisch

Federal Institute for Geosciences and Natural Resources,

Hannover, Germany
Appendix A: Historical earthquakes in the region and tables of used velocity models

Table 3 Historical earthquakes (1300-2000) with $I>$ $\mathrm{V}$ (or $M_{\mathrm{W}}>4$ ) in the region between HalleLeipzig and Vogtland/NW-Bohemia (Leydecker 2011; Grünthal and Wahlström 2012; Schwarz et al. 2010). $I$ is the epicentral intensity taken from LE, in case of missing or small values taken from $\mathrm{S} . M_{\mathrm{W}}$ is the transformed moment magnitude after $\mathrm{S}$ 
Table 3 Historical earthquakes (1300-2000) with $I_{0}>\mathrm{V}$ (or $M_{\mathrm{W}}>4$ ) in the region between Halle-Leipzig and Vogtland/NWBohemia (Leydecker 2011; Grünthal and Wahlström 2012; Schwarz et al. 2010). $I_{0}$ is the epicentral intensity taken from LE, in case of missing or small values taken from S. $M_{\mathrm{W}}$ is the transformed moment magnitude after $\mathrm{S}$

\begin{tabular}{|c|c|c|c|c|c|c|c|c|}
\hline date & $I_{0}$ & $M_{\mathrm{W}}$ & Lon $\mathrm{E}^{\circ}$ & Lat $\mathrm{N}^{\circ}$ & Location & $\mathrm{LE}$ & GR & $\mathrm{S}$ \\
\hline 1323 & $\mathrm{VI} 1 / 2$ & 4.6 & 12.34 & 51.10 & Grimma & $\mathrm{x}$ & & \\
\hline 1326 & $\mathrm{VI} 1 / 2$ & 4.6 & 12.20 & 50.80 & Gera & $\mathrm{x}$ & $\mathrm{x}$ & $\mathrm{x}$ \\
\hline $1332,12 \mathrm{Feb}$ & $\mathrm{V} 1 / 2$ & 4.0 & 12.20 & 50.80 & Gera & $\mathrm{x}$ & $\mathrm{x}$ & $\mathrm{x}$ \\
\hline 1540, 26 Jun 19:00 & $\mathrm{VI} 1 / 2$ & 4.6 & 12.90 & 51.10 & N.Sachsen & $\mathrm{x}$ & $\mathrm{x}$ & $\mathrm{x}$ \\
\hline 1552, 6 Mar & VI & 4.3 & 13.08 & 50.58 & Annaberg-Buchholz & $\mathrm{x}$ & $\mathrm{x}$ & $\mathrm{x}$ \\
\hline 1552, 20 Apr 09:00 & $\mathrm{V} 1 / 2$ & 4.0 & 12.66 & 50.57 & Schneeberg & $\mathrm{x}$ & $\mathrm{x}$ & $\mathrm{x}$ \\
\hline 1553, 17 Aug 19:30 & $\mathrm{VI} 1 / 2$ & 4.6 & 12.90 & 51.10 & Torgau & $\mathrm{x}$ & $\mathrm{x}$ & $\mathrm{x}$ \\
\hline $1568,26 \mathrm{Jul}$ & $\mathrm{V} 1 / 2$ & 4.0 & 13.05 & 51.12 & N-Sachsen & $\mathrm{x}$ & $\mathrm{x}$ & $\mathrm{x}$ \\
\hline 1578, 27 Apr 11:00 & $\mathrm{VI} 1 / 2$ & 4.6 & 12.23 & 50.88 & Gera & $\mathrm{x}$ & $\mathrm{x}$ & $\mathrm{x}$ \\
\hline 1598, 16 Dec 07:00 & $\mathrm{VI} 1 / 2$ & 4.6 & 12.18 & 50.87 & Gera & $\mathrm{x}$ & $\mathrm{x}$ & $\mathrm{x}$ \\
\hline $1616,18 \mathrm{Dec}$ & $\mathrm{V} 1 / 2$ & 4.0 & 12.25 & 51.20 & Leipzig & $\mathrm{x}$ & $\mathrm{x}$ & $\mathrm{x}$ \\
\hline 1674, Nov & $\mathrm{V} 1 / 2$ & 4.0 & 13.42 & 50.42 & Erzgebirge & $\mathrm{x}$ & & \\
\hline $1695,18 \mathrm{Apr}$ & $\mathrm{V} 1 / 2$ & 3.8 & 11.91 & 50.97 & Eisenberg & $\mathrm{x}$ & $\mathrm{x}$ & $\mathrm{x}$ \\
\hline 1701, 27 Mar 15:00 & $\mathrm{V} 1 / 2$ & 4.0 & 12.64 & 50.59 & Schneeberg & $\mathrm{x}$ & $\mathrm{x}$ & $\mathrm{x}$ \\
\hline 1701, 8 Apr 01:30 & $\mathrm{V} 1 / 2$ & 4.0 & 12.64 & 50.59 & Schneeberg & $\mathrm{x}$ & $\mathrm{x}$ & $\mathrm{x}$ \\
\hline 1711,25 Oct $19: 15$ & VI & 3.8 & 12.33 & 51.33 & Leipzig & $\mathrm{x}$ & $\mathrm{x}$ & $\mathrm{x}$ \\
\hline 1720, 1 Jul 17:00 & VI & 4.3 & 12.40 & 50.56 & Vogtland & $\mathrm{x}$ & $\mathrm{x}$ & $\mathrm{x}$ \\
\hline 1770, 4 Nov 01:00 & $\mathrm{V} 1 / 2$ & 4.0 & 12.43 & 50.25 & Kraslice (CZ) & $\mathrm{x}$ & $\mathrm{x}$ & $\mathrm{x}$ \\
\hline 1771, 6 Jan 16:00 & VI & 4.3 & 12.43 & 50.25 & Kraslice (CZ) & $\mathrm{x}$ & $\mathrm{x}$ & $\mathrm{x}$ \\
\hline 1789, 26 Aug 09:30 & $\mathrm{V} 1 / 2$ & 3.7 & 12.12 & 50.55 & Plauen & $\mathrm{x}$ & $\mathrm{x}$ & $\mathrm{x}$ \\
\hline 1811, 12 Dec 20:00 & $\mathrm{V} 1 / 2$ & 3.9 & 12.97 & 50.63 & Annaberg-Buchholz & & & $\mathrm{x}$ \\
\hline 1824, 13 Jan 13:00 & $\mathrm{V} 1 / 2$ & 4.0 & 12.51 & 50.33 & Kraslice (CZ) & $\mathrm{x}$ & $\mathrm{x}$ & $\mathrm{x}$ \\
\hline 1824, 19 Jan 16:30 & $\mathrm{V} 1 / 2$ & 3.8 & 12.40 & 50.20 & Vogtland & $\mathrm{x}$ & $\mathrm{x}$ & $\mathrm{x}$ \\
\hline 1850, 15 Jul 02:45 & $\mathrm{V} 1 / 2$ & 4.0 & 12.76 & 50.18 & Sokolov (CZ) & $\mathrm{x}$ & $\mathrm{x}$ & $\mathrm{x}$ \\
\hline 1857, 7 Jun 15:07 & $\mathrm{V} 1 / 2$ & 3.9 & 12.09 & 50.82 & $\mathrm{~S}$ of Gera & $\mathrm{x}$ & $\mathrm{x}$ & $\mathrm{x}$ \\
\hline 1862, 9 Jan $15: 54$ & VI & 4.3 & 12.22 & 50.04 & Cheb (Eger, CZ) & $\mathrm{x}$ & & \\
\hline 1872, 6 Mar 15:55 & VII & 5.2 & 12.28 & 50.86 & Posterstein/Gera & $\mathrm{x}$ & $\mathrm{x}$ & $\mathrm{x}$ \\
\hline 1875, 23 Nov 00:45 & $\mathrm{V} 1 / 2$ & 4.0 & 12.08 & 50.30 & Plauen & $\mathrm{x}$ & & $\mathrm{x}$ \\
\hline 1877,5 Oct $04: 30$ & $\mathrm{~V} 1 / 2$ & 3.8 & 13.66 & 50.80 & Erzgebirge & $\mathrm{x}$ & $\mathrm{x}$ & $\mathrm{x}$ \\
\hline 1883,20 Oct $22: 30$ & $\mathrm{~V} 1 / 2$ & 3.8 & 12.11 & 50.52 & Gera & $\mathrm{x}$ & & $\mathrm{x}$ \\
\hline 1888, 26 Dec 00:12 & $\mathrm{V} 1 / 2$ & 3.3 & 12.24 & 50.30 & Auerbach & $\mathrm{x}$ & $\mathrm{x}$ & $\mathrm{x}$ \\
\hline 1896, 16 Mai 20:50 & VI & 4.3 & 12.30 & 50.50 & Vogtland & & & $\mathrm{x}$ \\
\hline 1896, 3 Nov 21:10 & $\mathrm{V} 1 / 2$ & 4.1 & 13.50 & 50.59 & Erzgebirge & & & $\mathrm{x}$ \\
\hline 1897,25 Oct $21: 00$ & $\mathrm{~V} 1 / 2$ & 3.8 & 12.40 & 50.35 & Zwota & $\mathrm{x}$ & $\mathrm{x}$ & $\mathrm{x}$ \\
\hline 1897, 29 Oct 19:45 & $\mathrm{VI} 1 / 2$ & 4.2 & 12.48 & 50.35 & Klingenthal & $\mathrm{x}$ & $\mathrm{x}$ & $\mathrm{x}$ \\
\hline 1897, 7 Nov 04:45 & VI & 4.3 & 12.50 & 50.30 & Kraslice (CZ) & $\mathrm{x}$ & $\mathrm{x}$ & $\mathrm{x}$ \\
\hline 1897, 7 Nov 04:58 & $\mathrm{VI} 1 / 2$ & 4.2 & 12.48 & 50.35 & Klingenthal & $\mathrm{x}$ & $\mathrm{x}$ & $\mathrm{x}$ \\
\hline 1897, 17 Nov 06:30 & VI & 4.2 & 12.40 & 50.20 & Vogtland & $\mathrm{x}$ & $\mathrm{x}$ & $\mathrm{x}$ \\
\hline 1897, 17 Nov 07:43 & $\mathrm{V} 1 / 2$ & 4.0 & 12.32 & 50.22 & Kraslice (CZ) & & & $\mathrm{x}$ \\
\hline 1900,25 Jul 18:40 & $\mathrm{V} 1 / 2$ & 3.8 & 12.45 & 50.35 & Klingenthal & $\mathrm{x}$ & $\mathrm{x}$ & $\mathrm{x}$ \\
\hline 1903, 21 Feb 21:09 & VI & 4.1 & 12.47 & 50.34 & $\mathrm{~S}$ of Klingenthal & $\mathrm{x}$ & $\mathrm{x}$ & $\mathrm{x}$ \\
\hline 1903, 23 Feb 05:31 & $\mathrm{V} 1 / 2$ & 3.7 & 12.42 & 50.30 & SW of Klingenthal & $\mathrm{x}$ & $\mathrm{x}$ & $\mathrm{x}$ \\
\hline 1903, 25 Feb 23:11 & VI & 4.1 & 12.33 & 50.27 & $\mathrm{~N}$ of Bad Brambach & $\mathrm{x}$ & $\mathrm{x}$ & $\mathrm{x}$ \\
\hline
\end{tabular}


Table 3 (continued)

\begin{tabular}{|c|c|c|c|c|c|c|c|c|}
\hline date & $I_{0}$ & $M_{\mathrm{W}}$ & Lon $\mathrm{E}^{\circ}$ & Lat $\mathrm{N}^{\circ}$ & Location & LE & GR & $\mathrm{S}$ \\
\hline 1903, 5 Mar 00:50 & $\mathrm{V} 1 / 2$ & 3.5 & 12.20 & 50.86 & Markneukirchen & $\mathrm{x}$ & $\mathrm{x}$ & $\mathrm{x}$ \\
\hline 1903, 5 Mar 20:37 & $\mathrm{VI} 1 / 2$ & 4.5 & 12.42 & 50.37 & $\mathrm{~N}$ of Zwota & $\mathrm{x}$ & $\mathrm{x}$ & $\mathrm{x}$ \\
\hline 1903, 5 Mar 20:55 & $\mathrm{VI} 1 / 2$ & 4.6 & 12.42 & 50.37 & $\mathrm{~N}$ of Zwota & $\mathrm{x}$ & $\mathrm{x}$ & $\mathrm{x}$ \\
\hline 1903, 6 Mar 01:13 & $\mathrm{V} 1 / 2$ & 3.7 & 12.20 & 50.18 & Markneukirchen & $\mathrm{x}$ & $\mathrm{x}$ & $\mathrm{x}$ \\
\hline 1903, 6 Mar 04:57 & VI & 4.2 & 12.47 & 50.34 & $\mathrm{~S}$ of Klingenthal & $\mathrm{x}$ & $\mathrm{x}$ & $\mathrm{x}$ \\
\hline 1903, 6 Mar 12:59 & $\mathrm{V} 1 / 2$ & 4.0 & 12.33 & 50.27 & Landwüst & $\mathrm{x}$ & $\mathrm{x}$ & $\mathrm{x}$ \\
\hline 1903, 6 Mar 19:11 & $\mathrm{V} 1 / 2$ & 4.2 & 12.28 & 50.26 & SE of Bad Elster & $\mathrm{x}$ & $\mathrm{x}$ & $\mathrm{x}$ \\
\hline 1903, 7 Mar 05:00 & $\mathrm{V} 1 / 2$ & 4.0 & 12.48 & 50.37 & Klingenthal & $\mathrm{x}$ & $\mathrm{x}$ & $\mathrm{x}$ \\
\hline 1903, 7 Mar 05:01 & VI & 4.2 & 12.60 & 50.30 & Vogtland & & & $\mathrm{x}$ \\
\hline 1903, 8 Mar 06:22 & $\mathrm{V} 1 / 2$ & 3.8 & 12.50 & 50.35 & E of Klingenthal & $\mathrm{x}$ & $\mathrm{x}$ & $\mathrm{x}$ \\
\hline 1903, 27 Apr 16:08 & VI & 4.1 & 12.29 & 50.27 & E of Bad Elster & $\mathrm{x}$ & $\mathrm{x}$ & \\
\hline 1905, 17 Aug 03:21 & $\mathrm{V} 1 / 2$ & 4.0 & 12.38 & 51.35 & Leipzig & $\mathrm{x}$ & $\mathrm{x}$ & $\mathrm{x}$ \\
\hline 1908, 21 Oct 14:04 & $\mathrm{V} 1 / 2$ & 3.9 & 12.32 & 50.27 & Landwüst & $\mathrm{x}$ & $\mathrm{x}$ & \\
\hline 1908, 21 Oct 20:39 & VI & 4.2 & 12.29 & 50.28 & E of Bad Elster & $\mathrm{x}$ & $\mathrm{x}$ & \\
\hline 1908,22 Oct $21: 42$ & $\mathrm{~V} 1 / 2$ & 3.8 & 12.49 & 50.35 & Klingenthal & $\mathrm{x}$ & $\mathrm{x}$ & \\
\hline 1908, 3 Nov 12:01 & $\mathrm{V} 1 / 2$ & 3.8 & 12.27 & 50.23 & NW of Bad Brambach & $\mathrm{x}$ & $\mathrm{x}$ & \\
\hline 1908, 3 Nov 13:24 & VI & 4.2 & 12.31 & 50.23 & $\mathrm{~N}$ of Bad Brambach & $\mathrm{x}$ & $\mathrm{x}$ & \\
\hline 1908, 3 Nov 17:21 & $\mathrm{VI} 1 / 2$ & 4.5 & 12.47 & 50.34 & $\mathrm{~S}$ of Klingenthal & $\mathrm{x}$ & $\mathrm{x}$ & $\mathrm{x}$ \\
\hline 1908, 4 Nov 03:32 & VI & 4.1 & 12.49 & 50.36 & Bad Klingenthal & $\mathrm{x}$ & $\mathrm{x}$ & $\mathrm{x}$ \\
\hline 1908, 4 Nov 10:55 & $\mathrm{VI} 1 / 2$ & 4.6 & 12.47 & 50.34 & $\mathrm{~S}$ of Klingenthal & $\mathrm{x}$ & $\mathrm{x}$ & $\mathrm{x}$ \\
\hline 1908, 4 Nov 13:10 & $\mathrm{VI} 1 / 2$ & 4.6 & 12.47 & 50.34 & $\mathrm{~S}$ of Klingenthal & $\mathrm{x}$ & $\mathrm{x}$ & $\mathrm{x}$ \\
\hline 1908, 4 Nov 20:41 & VI & 4.3 & 12.37 & 50.28 & Wernitzgrün & $\mathrm{x}$ & $\mathrm{x}$ & $\mathrm{x}$ \\
\hline 1908, 6 Nov 04:35 & $\mathrm{VI} 1 / 2$ & 4.7 & 12.47 & 50.34 & $\mathrm{~S}$ of Klingenthal & $\mathrm{x}$ & $\mathrm{x}$ & $\mathrm{x}$ \\
\hline 1908, 12 Nov 11:31 & $\mathrm{V} 1 / 2$ & 4.1 & 12.40 & 50.40 & Vogtland & & & $\mathrm{x}$ \\
\hline 1908, 19 Dec 05:03 & $\mathrm{V} 1 / 2$ & 3.2 & 12.39 & 51.11 & Geringswalde & $\mathrm{x}$ & $\mathrm{x}$ & $\mathrm{x}$ \\
\hline 1911, 2 Apr 03:10 & $\mathrm{V} 1 / 2$ & 3.7 & 12.50 & 50.30 & Vogtland & & & $\mathrm{x}$ \\
\hline 1911, 4 Apr 00:30 & $\mathrm{V} 1 / 2$ & 3.4 & 12.40 & 50.30 & Vogtland & & & $\mathrm{x}$ \\
\hline 1914, 22 Apr 19:10 & $\mathrm{V} 1 / 2$ & 4.0 & 12.20 & 50.20 & Vogtland & & & $\mathrm{x}$ \\
\hline 1914, 27 Jun 01:44 & VI & 4.2 & 12.43 & 51.36 & Leipzig & $\mathrm{x}$ & $\mathrm{x}$ & $\mathrm{x}$ \\
\hline 1926, 28 Jan 16:57 & VI & 4.2 & 11.76 & 50.88 & Gera & $\mathrm{x}$ & $\mathrm{x}$ & $\mathrm{x}$ \\
\hline 1929, 15 May 08:45 & $\mathrm{V} 1 / 2$ & 3.8 & 12.40 & 50.40 & Vogtland & & & $\mathrm{x}$ \\
\hline 1985, 14 Dec 05:38 & $\mathrm{VI} 1 / 2$ & 4.0 & 12.39 & 50.15 & Novy Kostel (CZ) & & & $\mathrm{x}$ \\
\hline 1985, 20 Dec 16:36 & VI & 3.8 & 12.27 & 50.14 & Novy Kostel (CZ) & $\mathrm{x}$ & & $\mathrm{x}$ \\
\hline 1985, 21 Dec 10:04 & VI & 3.8 & 12.27 & 50.14 & Novy Kostel (CZ) & $\mathrm{x}$ & & $\mathrm{x}$ \\
\hline 1985, 21 Dec 10:16 & VII & 4.7 & 12.50 & 50.20 & Novy Kostel (CZ) & $\mathrm{x}$ & $\mathrm{x}$ & $\mathrm{x}$ \\
\hline 1985, 23 Dec 03:24 & $\mathrm{V} 1 / 2$ & 3.8 & 12.27 & 50.14 & Novy Kostel (CZ) & $\mathrm{x}$ & & $\mathrm{x}$ \\
\hline 1985, 23 Dec 04:27 & $\mathrm{VI} 1 / 2$ & 3.9 & 12.27 & 50.14 & Novy Kostel (CZ) & $\mathrm{x}$ & & $\mathrm{x}$ \\
\hline 1985, 24 Dec 00:04 & $\mathrm{V} 1 / 2$ & 4.0 & 12.45 & 50.24 & Novy Kostel (CZ) & $\mathrm{x}$ & $\mathrm{x}$ & $\mathrm{x}$ \\
\hline 1986, 20 Jan 23:38 & $\mathrm{VI} 1 / 2$ & 4.4 & 12.45 & 50.24 & Novy Kostel (CZ) & $\mathrm{x}$ & $\mathrm{x}$ & $\mathrm{x}$ \\
\hline 1986, 23 Jan 02:21 & $\mathrm{V} 1 / 2$ & 4.2 & 12.27 & 50.14 & Novy Kostel (CZ) & $\mathrm{x}$ & $\mathrm{x}$ & $\mathrm{x}$ \\
\hline
\end{tabular}

